\title{
SPATIAL HIGH-RESOLUTION MAPPING OF NATIONAL EMISSIONS
}

\author{
MARLENE S. PLEJDRUP, OLE-KENNETH NIELSEN \& HENRIK G. BRUUN \\ Department of Environmental Science, Aarhus University, Denmark
}

\begin{abstract}
Spatial distribution of emissions is a key element in assessing human exposure to air pollution through use of dispersion modelling. The quality of the spatial emission mapping is crucial for the quality, applicability and reliability of modelled air pollution levels, estimated human exposure, incurred health effects and related costs; all very important information for policy makers in decisions of implementation of environmental policies and measures. The purpose of the MapEIre project, funded by Ireland's Environmental Protection Agency, is to develop a high-resolution spatial mapping of the Irish emission inventory. The work is state-of-the-art and combines a large amount of statistical data with detailed spatial information to allow for a complete spatial emission mapping on a 1 kilometre by 1-kilometre resolution. The spatial model is developed as an integrated database system focusing on user-friendliness and performance optimisation. The spatial model for Ireland integrates official statistics, such as the Irish emission inventory, and censuses of population, housing and agriculture, with a large number of spatial datasets as diverse as heat demand, building use, road network and land cover maps, selected through a comprehensive assessment of available spatial data. The model covers 32 pollutants and 177 sectors and includes the entire Irish exclusive economic zone. The methodology developed and the lessons learned will be of great benefit to other countries, which are embarking to develop high-resolution spatial emission distributions. The detailed spatial distribution of emissions can be used by policy makers on both national and local level in decision making and prioritising of environmental measures. Further, it allows for a more detailed regulation, implementing measures targeting areas where emissions are highest, allowing for more cost-effective initiatives on local, regional and national scale.
\end{abstract}

Keywords: spatial emissions, emission mapping, emission inventory, GeoKey, air pollution, gridding.

\section{INTRODUCTION}

Human exposure to air pollution is a significant challenge to human health. WHO estimates that there were 3.7 million premature deaths in 2012 from urban and rural sources worldwide due to outdoor air pollution [1]. Results by Brandt et al. [2] show that outdoor air pollution caused a total number of 570.000 premature deaths in the year 2011 in Europe. While emissions in Europe are generally decreasing according to the European Environment Agency, EEA [3] there are still challenges at the local and regional scale that can only be quantified using high-resolution spatial models.

The impacts from air pollution are not equally distributed, but depend on geography, socio-demographic and socio-economic factors, including place of work and residence, age, gender, social status, and level of income. Including detailed spatial and temporal distributed emissions improve air quality modelling and contribute to understand the link between air pollution and distribution of related health impacts by adding knowledge that influences modelling of human exposure levels.

Air pollution, in general, is a transboundary and scale dependent challenge with global, regional, national and local sources giving rise to large geographic variability and thereby large differences in the geographical distribution of human exposure to air pollution. Therefore, the lifetime exposure and personal risk factors for mortality and morbidity outcomes due to air pollution, and thereby their welfare, is very dependent on the individual's possibilities to live in a clean environment. 
Spatial distribution of emissions is a key element in assessing human exposure to air pollution through the use of dispersion modelling. The quality of the spatial emissions distribution is crucial for the quality, applicability and reliability of the modelled air pollution levels, the estimated human exposure, incurred health effects and related costs; all issues that are very important information for policy makers in decisions of implementation of environmental policies and measures.

Several studies have endeavoured to make a spatial distribution for the whole of Europe. These include the EDGAR system [4] developed by the European Commission Joint Research Centre and the system developed by TNO [5]. In general, both of these projects used point source information, where it was available, but otherwise relied on very general proxies such as population density or land-use information. The point source information came from international databases such as EPER (European Pollutant Emission Register) and the World Electric Power Plants Database. For the area sources, both studies used very crude spatial proxies to distribute emissions, such as population density, FAO (the Food and Agriculture Organization) data on distribution of livestock, and CORINE (Coordination of Information on the Environment) land cover. With thorough research at the country level, it is possible to significantly improve the spatial distribution compared to these models attempting to cover the entire European continent.

Few studies have covered the whole range of pollutants or sectors, in fact most of the published studies have focussed on single pollutants [6]-[9] or single sectors [10]-[15]. In addition, many studies have not been at the national level but at a city or regional level [16] [18]. A few studies have focused on spatial distribution for a range of pollutants at the national level for all emission sectors [19]-[21].

One of the primary objectives of the MapEIre project is to develop a high-resolution spatial emission model for Ireland that will allow for a more detailed regulation implementing measures targeting areas where the emissions are highest allowing for more cost-effective initiatives. Development of a national spatial emission model covering the entire country allows for assessment of effects of measures on local, regional and national scale.

\section{METHODOLOGY}

The integrated spatial emission mapping model for Ireland, MapEIre, is developed with the aim to use the most detailed data available regarding information on both emissions and spatial conditions, while still being easy to update as new knowledge becomes available.

The model is built as an integrated database system, aiming at being easy to operate for the end users, but still able to handle the large data amounts and run complex calculations at high speed. This is ensured through a well-organised setup, a clear data concept, and precise interrelationships between the incorporated parameters, e.g. via use of primary keys. The built-in simple user interfaces minimise the risk of introduction of errors by the user. The model includes all pollutants in the Irish emission inventory as reported to the Convention on Long-Range Transboundary Air Pollution, CLRTAP, for air pollution and to the United Nations Framework Convention on Climate Change, UNFCCC, for greenhouse gases. The sectoral levels in MapEIre follow the sectors in the corresponding reporting tables (Nomenclature for Reporting, NFR, and Common reporting format CRF). In total MapEIre covers 32 pollutants and 177 sectors. The model is linked directly to the data in the reporting tables, making it easy to update as recalculations are made in the emission inventory, and ensuring consistency of emission totals in the national and the spatial inventory.

The spatial resolution in MapEIre is a $1 \mathrm{~km} \times 1 \mathrm{~km}$ orthogonal grid following the 1000meter x-axis and y-axis in the TM65/Irish Grid projection (EPSG 29902). The allocation of the national total emissions is based on a large number of spatial distribution keys (GeoKeys), 
which are normalized tables holding the shares of the national total emission for a given sector and a given pollutant, to be allocated to the individual cells in a pre-defined grid.

The national emission inventories prepared by Ireland's Environmental Protection Agency (EPA), including associated detailed background data, comprise the most accurate and detailed data source regarding emissions. For the sources that are included in the national emission inventory as point sources, the GeoKeys are based on the data available in the inventory (emissions, fuel consumption, activity level and/or geographical location). Annual plant specific emissions data result in the most accurate mapping but are available to a limited degree. The European Pollutant Release and Transfer Register (E-PRTR) holds data for plants in e.g. the energy and industrial sector that exceeds specified emission thresholds and Emission Trading Scheme (ETS) data contains activity data and $\mathrm{CO}_{2}$ emissions. E-PRTR data and ETS data have been used to prepare the GeoKey for the public electricity and heat production sector, which is covered only by point source emissions (Fig. 1). Plant specific emissions data provide the opportunity to prepare pollutant specific GeoKeys. When plant specific emissions are not available, fuel consumption data is used to prepare the GeoKeys, which in that case apply for all pollutants. If the location of the point sources in a sector are available without further details, GeoKeys are created to distribute the emissions evenly between the address points.

Spatial proxy data have to be used for emission mapping for sectors without plant specific data. A comprehensive assessment of the available spatial data is crucial to ensure the highest accuracy of the resulting spatial emissions. Many sources in the emission inventory are area sources, which refer to emissions that come from small point sources that are too large in number to be treated individually such as households, from line sources like roads and railways, or from area sources like fields or other areas with common land use. GeoKeys for area sources distribute the emissions to the relevant points, lines or areas according to the share of total number, length or area.

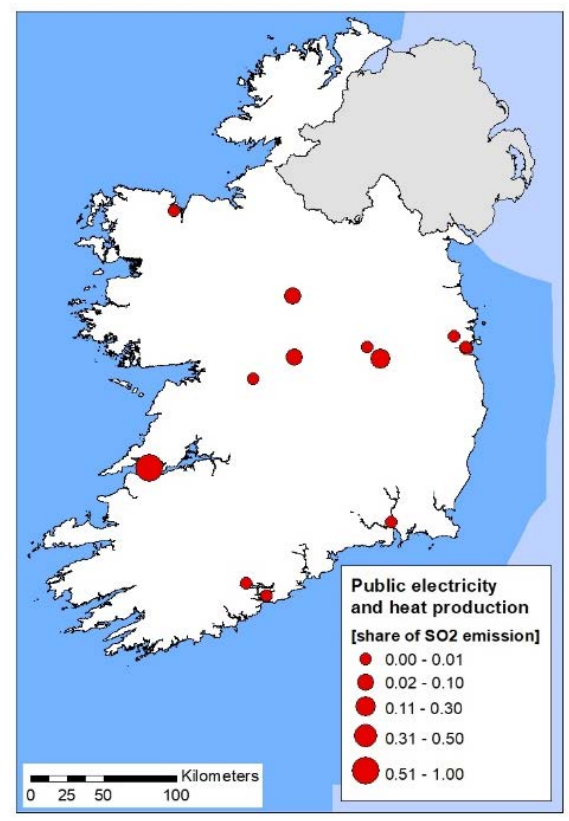

Figure 1: GeoKey for $\mathrm{SO}_{2}$ emissions from public electricity and heat production. 


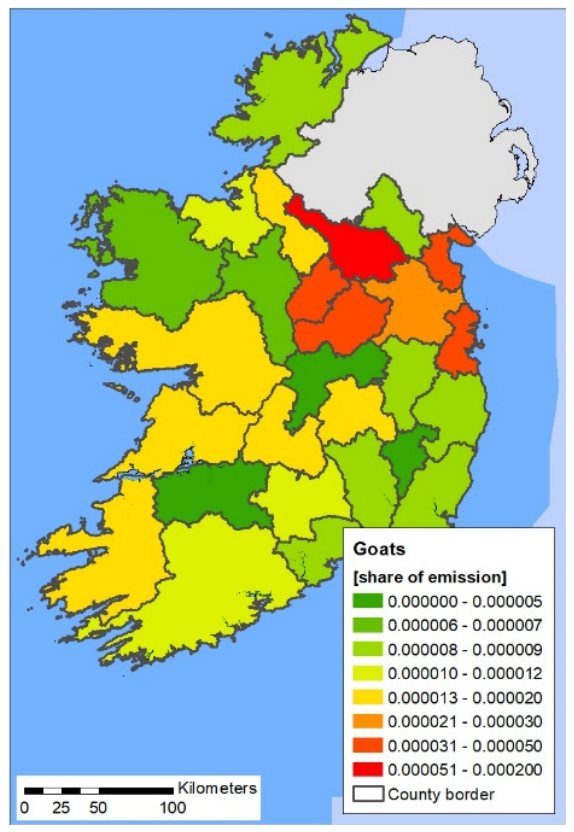

(a)

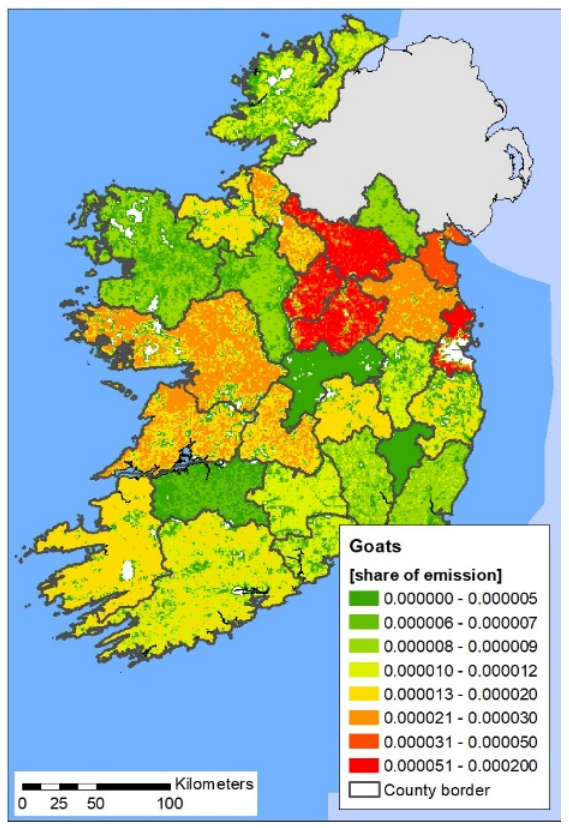

(b)

Figure 2: GeoKey for manure management and enteric fermentation for goats based on animal numbers by county distributed. (a) Evenly in the county; (b) According to location of farms.

Official statistics, such as census of population, housing and agriculture, provide useful input for generating GeoKeys. Statistics are often available on an aggregated spatial level following administrative units, e.g. electoral districts or counties. In such a case land use data can be used to improve the spatial emission distribution by including relevant areas only, e.g. exclude urban areas from the GeoKey for open burning of waste so that it only allocate emissions to rural residential buildings or allocate emissions from animals to farm areas only. The latter is shown in Fig. 2 where it is clearly visible that areas without agricultural activities would be overestimated.

A number of sectors is made up of a combination of point and area sources, and the GeoKeys are prepared by combining a GeoKey for the point sources, e.g. large plants in the food and beverages industry based on plant specific fuel consumption, and a GeoKey for the area sources, e.g. allocation according to the heat demand in industrial buildings (Fig. 3).

In this case, the share of the sectoral emissions to be mapped according to the point source GeoKey follow the share of the total sectoral fuel consumption allocated to the point sources. In many cases, the most accurate spatial representation is made by combining several statistical and spatial datasets.

\section{RESULTS AND DISCUSSION}

Based on the model, emissions have been mapped on $1 \mathrm{~km} \times 1 \mathrm{~km}$ for all pollutants and all sectors, and the output is available as emission maps that can be downloaded from the project website (www.mapeire.dk) as pictures (jpg files) and digital maps (shape files) for use in Geographical Information Systems, GIS. 


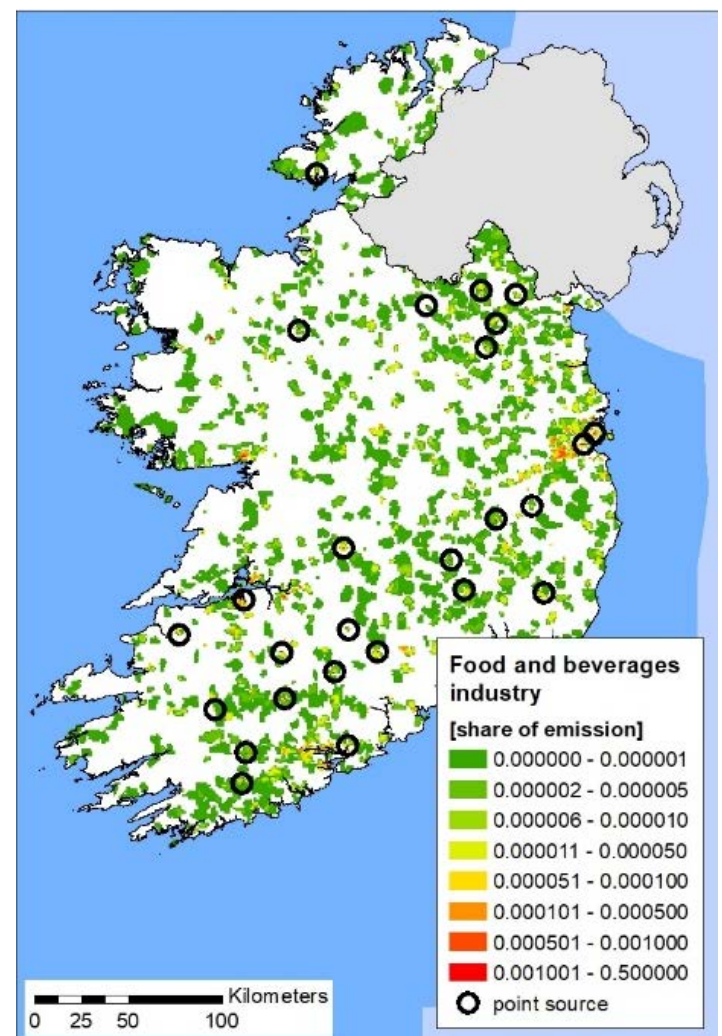

Figure 3: GeoKey for food and beverages industry.

Fig. 4 show the resulting spatial national total $\mathrm{NO}_{\mathrm{x}}$ and $\mathrm{PM}_{2.5}$ emission from the MapEIre model. The spatial patterns vary due to different sectoral contribution for different pollutants. The largest sources to emissions of $\mathrm{NO}_{\mathrm{x}}$ are road transport, industry, power plants, residential plants and other mobile sources, and correspondingly the map is dominated by point sources in the energy and industrial sector, major roads and urban areas. The largest sources to emissions of $\mathrm{PM}_{2.5}$ are residential homes, road transport, agriculture and industry, and hence urban, agricultural and industrial areas are visible on the map. The road network is not apparent on the map due to the large emissions from residential homes and agriculture covering most of the land area.

Different characteristics should be considered in the selection of which spatial data to include in the GeoKey preparation; coverage, accuracy and applicability.

The spatial data should preferably cover the entire domain of the spatial model to avoid lack of emissions in uncovered areas. An exception to this rule of thumb is made for road transport, where detailed mileage data are available for the national road network (NR), covering primary and secondary roads, including information on share of heavy vehicles, and representing around $45 \%$ of the national total mileage. The GeoKey for road transport is created as a combination of a GeoKey for NR and a GeoKey for other roads, where the remaining part of the national total mileage is allocated to the road network for other roads than NR according to the share of the total road length for the individual grid cells. The 


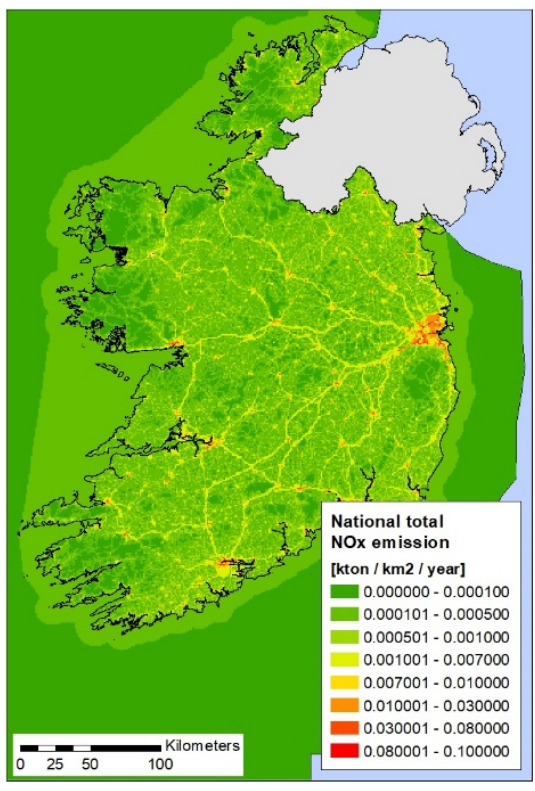

(a)

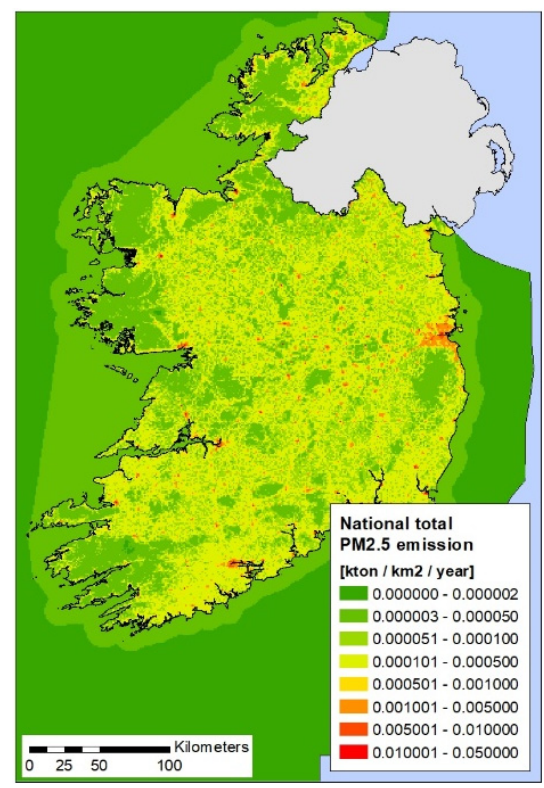

(b)

Figure 4: National total emissions. (a) $\mathrm{NO}_{\mathrm{x}}$; and (b) $\mathrm{PM}_{2.5}$.

GeoKey for passenger cars road transport is shown in Fig. 5(a). For comparison Fig. 5(b) show a GeoKey based solely on the location of the road network, leading to a more even distribution leading to underestimation of emissions on heavily trafficked roads.

The accuracy depend on the correctness of the spatial information i.e. the uncertainty level, the update frequency, and the time for the latest update. The correctness applies to both the spatial location and the attribute information. Spatial data on buildings from GeoDirectory are very accurate regarding the location of the individual buildings, while the building use categorisation are less accurate as some buildings fall into more categories or the building use is unknown. The agricultural census holds animal numbers aggregated on administrative units (electoral district or county). To improve the spatial allocation of emissions from animals, the census data is combined with the Land Parcel Identification System (LPIS), and the GeoKeys are calculated as a combination of the share of animals by administrative unit and share of the relevant LPIS areas by grid cell for the individual administrative units (Fig. 2).

The applicability is in this case the usefulness of a spatial data set as a proxy for an emission allocation. A spatial data set of high accuracy might not be a good proxy and vice versa. Population density based on detailed national statistics is an example of spatial data of high accuracy with low uncertainty level, high update frequency, and full geographical coverage, but a poor proxy for many sectors. In lack of sector specific spatial data, population density can be the best available proxy. Depending on the emission sector and the local circumstances, use of population density might lead to overestimation in densely populated areas, e.g. when used as a proxy for residential wood burning, which might be less common in city centres where the population density is highest. Fig. 6 shows the GeoKeys for $\mathrm{PM}_{2.5}$ emissions from stationary combustion in the residential sector and for population density. The maps visualise the risk of overestimation of emissions in urban areas if population 


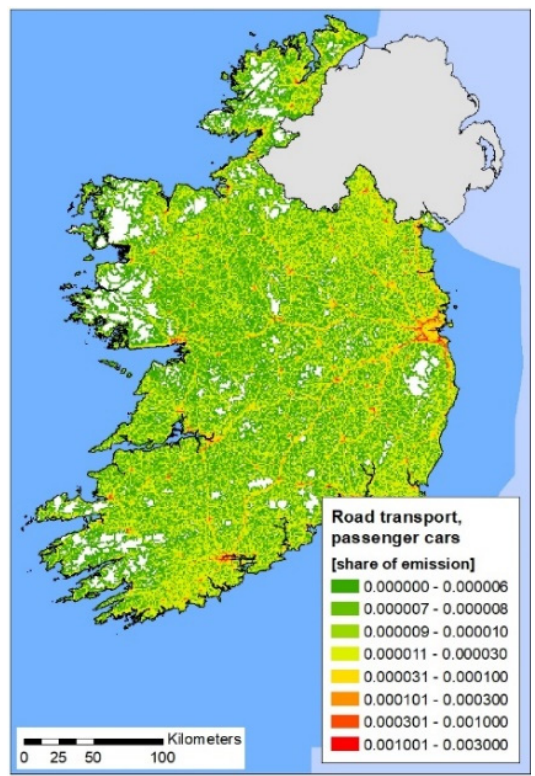

(a)

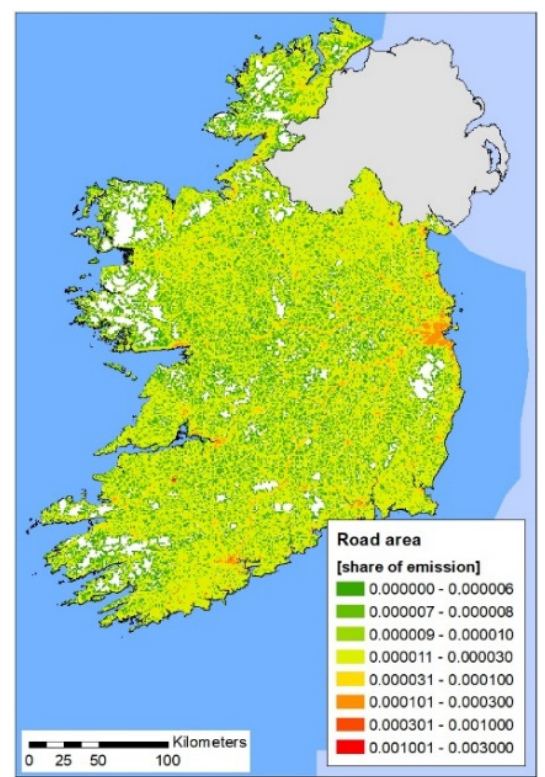

(b)

Figure 5: GeoKey for road transport with passenger cars. (a) Mileage data for national roads and other roads network for the remaining part of the total mileage; and (b) Road network.

density is used to allocate emissions instead of the sector specific GeoKey based on data on primary heating for all dwellings from the census from the Central Statistics Office and average fuel use from the energy statistics. Simply using population density would overestimate emissions around the major urban areas and this error would be magnified when calculating human exposure, related health effect and costs. This highlights the need for combining multiple spatial and statistical datasets rather than using simple spatial proxies.

The poorest proxies in the MapEIre model are GeoKeys that allocate emissions evenly to the land area and the total domain area, respectively. These are only applied to few minor sources and to emissions from international navigation and aviation, which, due to reporting requirements, must be allocated to the national domain even though the emissions partly occur outside the domain.

In general, local or national data are preferable, but international data can be used as proxy, even though the accuracy is most likely lower. MapEIre include data from CORINE Land Cover (cropland, forestland, grassland, settlements, wetland and other land) and Open Street Map (point data for hospitals and clinics) for making GeoKeys for the LULUCF sector and for $\mathrm{N}_{2} \mathrm{O}$ from anaesthesia use.

\section{CONCLUSIONS}

In this work, a detailed spatial emission inventory at a $1 \mathrm{~km} \times 1 \mathrm{~km}$ resolution has been developed. It covers all major pollutants for which there is a requirement to estimate and report emissions. In contrast to many other studies, the sectoral level in this study is highly detailed, and by including a much more detailed sectoral breakdown, it has been possible to use more detailed GeoKeys and hence get a much more accurate spatial distribution. 


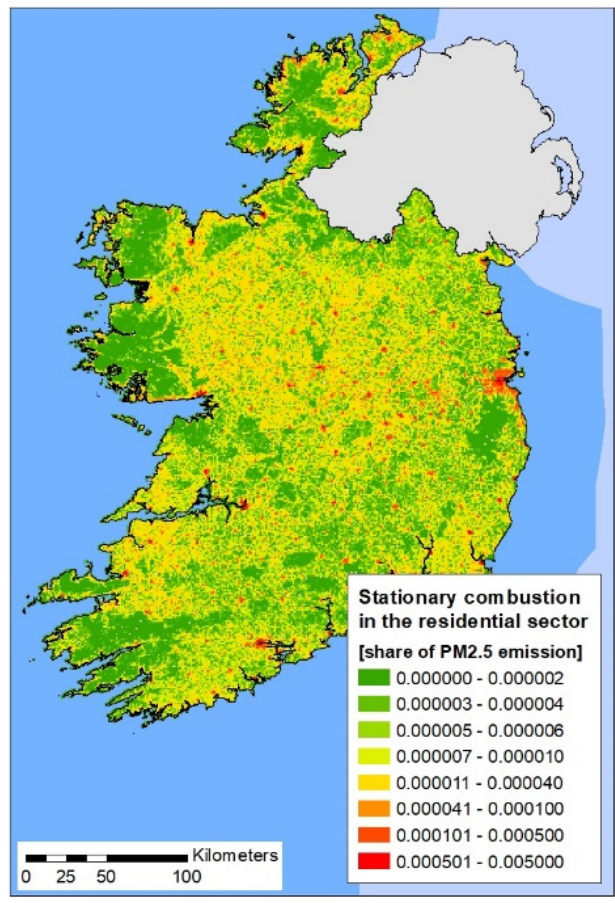

(a)

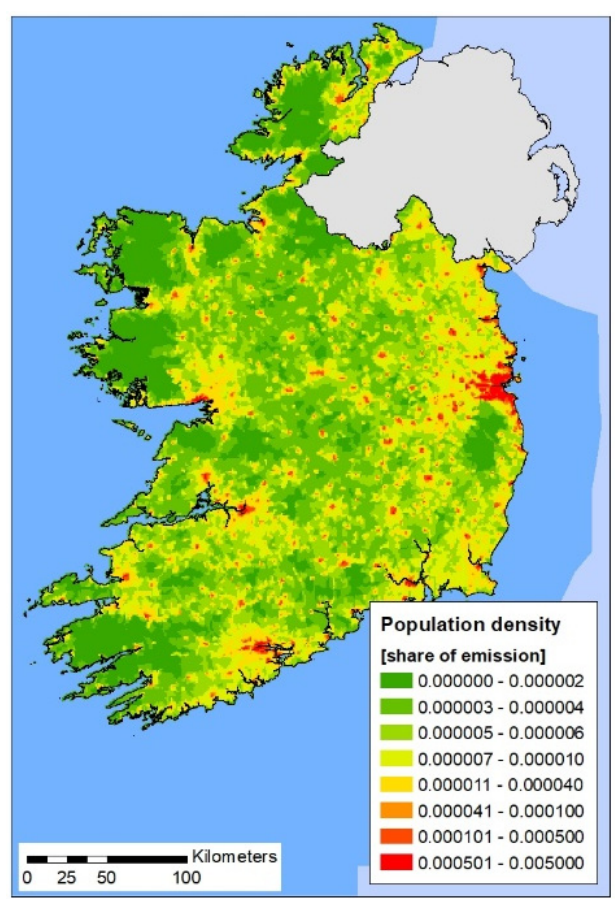

(b)

Figure 6: GeoKeys. (a) $\mathrm{PM}_{2.5}$ from stationary combustion in the residential sector; and (b) Population density.

The results of the project (Fig. 4) clearly shows the hotspots of emissions, which will make it possible to target measures in areas of the country, where it will have the most effect and it forms a solid foundation to further assess the human exposure and the deposition of harmful substances to vulnerable nature. The outcome of this work will also support further research and development of an air quality modelling framework in Ireland and can be used internationally to improve the general knowledge of spatial distribution of emissions.

The methodology used in this study can serve as an example for other countries that are starting to make a high-resolution nationwide mapping. The identification of all available spatial data is paramount and this study shows that in many cases several spatial datasets have to be combined in order to achieve the most accurate result.

As alluded to in the discussion there is still room for improving the spatial inventory both related to the development of better GeoKeys, but also in the selection of the most suitable GeoKey to a given emission source. In addition, it is important to note that the current GeoKeys have been elaborated for 2015 and an important next step will be to develop timeseries, so that the changes in spatial emission patterns over time can be reflected in the model.

\section{ACKNOWLEDGEMENTS}

This work has been funded by Ireland's Environmental Protection Agency Research Call 2015 (Ref: 2015-CCRP-MS.26).

The authors would like to thank Paul Duffy, Bernard Hyde and Ann Marie Ryan from the emission inventory team at Ireland's Environmental Protection Agency (EPA) for the access 
to detailed data from the emission inventory as well as valuable discussions on data and national circumstances. Also, the authors would like to thank David Kelleghan for providing highly detailed data on pigs and poultry houses.

The authors would like to acknowledge the advice and help provided by members of the Steering Committee of the project.

\section{REFERENCES}

[1] 7 million premature deaths annually linked to air pollution. World Health Organization. Online. http://www.who.int/mediacentre/news/releases/2014/airpollution/en/. Accessed on: 14 Feb. 2018.

[2] Brandt, J. et al., Contribution from the ten major emission sectors in Europe and Denmark to the health-cost externalities of air pollution using the EVA model system - an integrated modelling approach. Atmospheric Chemistry and Physics, 13(15), pp. 7725-7746, 2013.

[3] European Environment Agency (EEA), European Union emission inventory report 1990-2015 under the UNECE Convention on Long-range Transboundary Air Pollution (LRTAP). EEA Report No 9/2017, 2017.

[4] EDGAR - Emissions Database for Global Atmospheric Research. European Commission. Online. http://edgar.jrc.ec.europa.eu/index.php. Accessed on: 14 Feb. 2018 .

[5] Umweltbundesamt, A high resolution European emission data base for the year 2005. 2013.

[6] Bo, Y., Cai, H. \& Xie, S.D., Spatial and temporal variation of historical anthropogenic NMVOCs emission inventories in China. Atmos. Chem. Phys., 8, pp. 7297-7316, 2008.

[7] Sahu, S.K., Beig, G. \& Parkhi, N.S., Emissions inventory of anthropogenic $\mathrm{PM}_{2.5}$ and $\mathrm{PM}_{10}$ in Delhi during Commonwealth Games 2010. Atmospheric Environment, 45, pp. 6180-6190, 2011.

[8] Dalvi, M., Beig, G., Patil, U., Kaginalkar, A., Sharma, C. \& Mitra, A.P., A GIS based methodology for gridding of large-scale emission inventories: Application to carbonmonoxide emissions over Indian region. Atmospheric Environment, 40, pp. 29953007, 2006.

[9] Wu, S.-P., Zhang, Y.-J., Schwab, J.J., Li, Y.-F., Liu, Y.-L. \& Yuan, C.-S., Highresolution ammonia emissions inventories in Fujian, China, 2009-2015. Atmospheric Environment, 162, pp. 100-114, 2017.

[10] Skjøth, C.A. et al., Spatial and temporal variations in ammonia emissions - a freely accessible model code for Europe. Atmos. Chem. Phys., 11, pp. 5221-5236, 2011.

[11] Hellsten, S. et al., Modelling the spatial distribution of ammonia emissions in the UK. Environmental Pollution, 154, pp. 370-379, 2008.

[12] Puliafito, S.E., Allende, D., Pinto, S. \& Castesana, P., High resolution inventory of GHG emissions of the road transport sector in Argentina. Atmospheric Environment, 101, pp. 303-311, 2015.

[13] Plejdrup, M.S., Nielsen, O.-K. \& Brandt, J., Spatial emission modelling for residential wood combustion in Denmark. Atmospheric Environment, 144, pp. 389-396, 2016.

[14] Johansson, L., Jalkanen, J.-P. \& Kukkonen, J., Global assessment of shipping emissions in 2015 on a high spatial and temporal resolution. Atmospheric Environment, 167, pp. 403-415, 2017. 
[15] Castesana, P., Dawidowski, L., Finster, L., Gomez, D. \& Taboada, M., Ammonia emissions from the agriculture sector in Argentina; 2000-2012. Atmospheric Environment, to be published.

[16] Guttikunda, S.K. \& Calori, G., A GIS based emissions inventory at $1 \mathrm{~km} \mathrm{x} 1 \mathrm{~km}$ spatial resolution for air pollution analysis in Delhi, India. Atmospheric Environment, 67, pp. 101-111, 2013.

[17] Tian, Y.Q., Radke, J.D., Gong, P. \& Yu, Q., Model development for spatial variation of $\mathrm{PM}_{2.5}$ emissions from residential wood burning. Atmospheric Environment, 38, pp. 833-843, 2004.

[18] Qi, J., Zheng, B., Li, M., Yu, F., Chen, C., Liu, F., Zhou, X., Yuan, J., Zhang, Q. \& He, K., A high-resolution air pollutants emission inventory in 2013 for the BeijingTianjin-Hebei region, China. Atmospheric Environment, 170, pp. 156-168, 2017.

[19] Kannari, A., Tonooka, Y., Baba, T. \& Murano, K., Development of multiple-species $1 \mathrm{~km} \times 1 \mathrm{~km}$ resolution hourly basis emissions inventory for Japan. Atmospheric Environment, 41, pp. 3428-3439, 2007.

[20] Tsilingiridis, G., Sidiropoulos, C., Pentaliotis, A., Evripidou, C., Papastavros, C., Mesimeris, T. \& Papastavrou, M., A spatially allocated emissions inventory for Cyprus. Global NEST Journal, 12(434), pp. 99-107, 2010.

[21] Plejdrup, M.S. \& Gyldenkærne, S., Spatial distribution of emissions to air - the SPREAD model. National Environmental Research Institute, Aarhus University, Denmark, 72 pp. - NERI Technical Report no. 823, 2011. 